\title{
Correlates of keeping post-discharge appointments at a transitional care center: Implications for medical care disparities
}

\author{
Henry Carretta*1, Seyfullah Tingir ${ }^{2}$, Cara Pappas ${ }^{3}$, Amy L. $\mathrm{Ai}^{4}$ \\ ${ }^{1}$ Department of Behavioral Sciences and Social Medicine, Florida State University, United States \\ ${ }^{2}$ College of Education, Florida State University, United States \\ ${ }^{3}$ College of Nursing, Florida State University, United States \\ ${ }^{4}$ Colleges of Social Work and Nursing, Departments of Behavioral Science and Social Medicine and Psychology, Florida State \\ University, United States
}

Received: January 10, 2017

Accepted: February 19, 2017

Online Published: March 6, 2017

DOI: $10.5430 /$ jha.v6n2p53

URL: https://doi.org/10.5430/jha.v6n2p53

\begin{abstract}
Objective: Over the past decade, transitional care (TC) programs have demonstrated initial benefits for decreased care costs, reduced rehospitalizations and emergency department visits via care coordination team activities. Patients who completed their TC follow-up appointment subsequently have less frequent ER visits. The current study addressed correlates of missed appointments in a sample of under-investigated, mostly vulnerable (e.g., middle-aged, uninsured) patients.

Methods: We conducted a retrospective observational study of an appointment database for patients enrolled at a major transition center during the first three years since its establishment in Northern Florida. Patients $(\mathrm{n}=2,146)$ were referred to a Transitional Care Center (TCC) from a regional medical center after discharge. The type of insurance and demographic characteristics of the patients was used to predict missed appointments.

Results: Logistic regression analyses indicated that privately insured patients were more likely and Black publicly and privately insured patients were less likely to keep first appointments. No effect on keeping appointments was seen for uninsured patients. Conclusions: Our findings suggest that an appointment referral is a necessary but not sufficient step for the accomplishment of TC goals. Medical teams need to collaborate with other health professionals, such as social workers, to identify the barriers to keeping appointments and ensure effective solutions for achieving the goal of preventing future ER visits and rehospitalization.
\end{abstract}

Key Words: Transitional care, Missed appointments, Health insurance, Uninsured, Interprofessional collaboration

\section{INTRODUCTION}

Providing continuity of care across different health system environments is essential for high-quality patient care. ${ }^{[1,2]}$ Transitional care (TC) is defined as time-limited, interdisciplinary services designed to ensure health care continuity through timely transfer of patients from one level of care to another or from one type of setting to another for avoiding preventable poor outcomes among at-risk populations. ${ }^{[3-5]}$ TC services are aimed at avoiding preventable rehospitalizations (RH) and emergency room (ER) over-utilization, ultimately resulting in lower costs. ${ }^{[6]}$ Over the past decade, evidence has shown that TC programs encompass continuity

\footnotetext{
*Correspondence: Henry Carretta; Email: henry.carretta@med.fsu.edu; Address: Department of Behavioral Sciences and Social Medicine, Florida State University, United States.
} 
via multidisciplinary and interagency care coordination team activities, demonstrating initial benefits for decreased health care costs, reduced RH and ER visits, and increased patient satisfaction. ${ }^{[7-13]}$

Coordinating care through effective transitions thereby ensures care continuity for the most vulnerable patients afflicted with multiple chronic diseases. ${ }^{[10]}$ Recently, TC Management has been recognized as a reimbursable service in the 2013 Medicare Physician Fee Schedule. ${ }^{[14]}$ In 2011, TC leading researchers reviewed 21 randomized controlled trials (RCT) on TC interventions and found positive effects on $\mathrm{RH}$ in nine programs within the review. ${ }^{[6]}$ Most importantly, a new study found that patients who completed their TC follow-up appointment had less frequent ER visits in the year following their discharge. ${ }^{[8]}$ Despite many TC innovations and success stories, several gaps exist in this emerging research area, especially regarding the key factor, patient adherence with keeping the first follow-up appointment after their hospitalization.

First, there has been limited scientific investigation to inform implementation of TC for one of the most vulnerable populations (e.g., middle-aged chronic disease patients with limited health care access). Indeed, the first round of penalties from the Readmission Reduction Program within the Affordable Care Act showed that hospitals caring for the sickest, poorest, and most vulnerable patients often have exceptionally high $\mathrm{RH}$ rates and were more likely to incur such penalties. ${ }^{[15]}$ Second, these vulnerable patients tended to be frequent visitors to the ER even after keeping follow-up appointments. Follow-up after discharge from the ER is a critical contributor to reducing ER revisits. ${ }^{[8]}$ However, little is known about key predictors for keeping follow-up visits in this population. Finally, few studies have examined whether insurance status may play a role in keeping or missing appointment referral appointments to TC centers within this vulnerable population.

To meet these gaps, the present study conducted a retrospective record analysis for a sample of such patients referred to a major regional TC center during hospitalization. The region has a large community of minorities (e.g., African Americans and Latino Americans) and/or poor populations. The Transitional Care Center (TCC) of the Tallahassee Memorial Hospital (TMH) opened in 2011. The TCC enables outpatient follow-up and help to this population in establishing a medical home, addressing social barriers to care and lack of insurance coverage. The population served is mainly uninsured, middle-aged adults with multiple chronic diseases who lack a regular source of primary care. A large proportion of these patients are minorities. An estimated $30 \%$ of patients miss their TCC appointments - a major barrier to the care and health of patients according to an internal report of TMH in 2012. ${ }^{[16]}$

The goal of this preliminary study is to examine the characteristics of patients referred to the TCC in the first three years of operation to identify person-level characteristics associated with missing the first follow-up appointment. Based on the literature, we hypothesized that a racial minority status, especially Blacks, and uninsured status would be associated with less likelihood of keeping the first appointment. We also examined the moderation of insurance types on the link between race and keeping appointment via interaction analyses.

\section{METHOD}

\subsection{Sample and procedure}

To comply with HIPAA regulations, the third author deidentified appointment data provided by the parent hospital. We analyzed the electronic data (appointment database) on hospital patients referred to the TCC upon discharge from February 2011 to February 2014. The three-year TCC appointment database holds over 8,000 patient appointment records and 2,146 unique persons over time but our analysis was limited to each patient's first appointment. Our analysis was further limited to observations with no missing values. There were 297 patients with missing race, ethnicity or marital status values. These were excluded leaving 1,850 unique persons in the analysis.Appointment data were merged with data provided by the hospital and include: demographic variables for age, gender, race, ethnicity, marital status and health insurance type and status.

\subsection{Measures}

Categorical variables were coded as follows: (1) gender ( 0 = male, $1=$ female), ( 2$)$ race $(1=$ Black, $2=$ other, 3 = white), (3) ethnicity ( $1=$ Hispanic, $0=$ non-Hispanic), (4) marital status ( $1=$ married, $0=$ not married); and (5) health insurance status $(1=$ private, $2=$ public, and $3=$ self-pay). Age was left as a continuous variable. The outcome of interest was first appointment condition $(1=$ missed, $0=\mathrm{kept}$ ).

The criteria for referral to the TCC include: (1) no medical insurance; (2) three or more hospital admissions in the past 12 months; (3) no primary care provider; or (4) patient has a primary care provider (with or without insurance), but cannot get a follow-up appointment within seven days of discharge. There is no charge for the services provided by the TCC.

The analysis began with univariate statistics for all demographic and insurance type measures. We then conducted 
2-way analysis of all of our predictor variables by binary appointment condition and used a $t$-test or chi-squared test to identify statistically significant differences in predictor variables between the records with "missed" versus "kept" first appointments. Additional indicators were created for race and insurance categorical variables as well as interaction terms for race by ethnicity and race by insurance payment type.

We further investigated the relationship between our predictors and whether a patient missed or kept their first appointment by a series of nested logistic regression models in which the outcome was missed versus kept appointment. Model 1 included demographic and other predictors excluding payment indicators. Model 2 included Model 1 variables and payment indicators. Model 3 included all of the variables in Model 1 and 2 plus interaction terms for race by payment type. The final model (M3) included continuous age plus dummy variables:

Apt. Condition $=$ constant $+[$ age + female + Black + white + Hispanic + marital status + Black-Hispanic + other-Hispanic] + [private insurance + public insurance $]+[$ Black-public + Black-private + other-public + other-private $]+$ error

Robust estimation was used to account for unequal variance across the population. Likelihood ratio tests were used to identify the best fitting models. Stata ${ }^{\circledR}$ version 11 was used for analysis. Finally, since this is an early stage exploratory research we set our $p$-value for a two-sided test of significance equal to .10 .

\section{RESUltS}

\subsection{Descriptives}

Table 1 summarizes the demographics and insurance information for the sample of 1,850 first appointment records. No difference in mean age in the two groups was found. The overall gender pattern was balanced (female $=51 \%$; male $=49 \%$ ) and no difference was seen by appointment condition. The racial distribution between groups was more diverse and approached statistical significance $(p=.11)$. Overall, $41.1 \%$ of the study population was Black, $54.3 \%$ were white, and $4.6 \%$ were other race. With respect to ethnicity, no difference was seen by appointment condition. Overall, 3.6\% reported themselves as Hispanic, 96.4\% reported themselves non-Hispanic. Most patients (74.8\%) were unmarried. No difference was found in marital status by appointment condition. The majority of the sample overall was uninsured $(61.6 \%)$ and $13.1 \%$ had private insurance, and $25.2 \%$ had some type of public coverage from Medicare, Medicaid, or the Veterans Health Administration. Differences by appointment condition were significant $(p=.009)$. Private managed care companies were heavily represented in both the public Published by Sciedu Press and private insurance companies (data not shown). Lastly, with respect to first appointment status, 463 (25\%) of patients missed their first appointment, whereas 1,607 (75\%) patients kept their first appointment.

\subsection{Nested logistic regression models of (M1) age, gen- der, race, ethnicity, marital status and race by eth- nicity, (M2) M1 variables plus payment type, and (M3) M2 variables plus race by payment type on ap- pointment status}

Table 2 presents the results of our three logistic models. Age, gender and marital status were not significant in any of the three models. Black race was associated with increased odds of a missed appointment but was not significant $(p=.11)$ in Model 1. Other race was associated with a strong protective effect ( $p=.023$ ) against missed appointments, however the confidence intervals are quite wide so note the direction of the effect without giving it too much credence. Likewise, Hispanic ethnicity was strongly protective of missed appointments $(p=.08)$ but the wide confidence intervals mean the odds ratio is measured with low precision just like the other race category. The Black Hispanic and other Hispanic racial/ethnic groups have very strong associations with missed first appointment but again their precision is low. The low precision of estimates for other race and Hispanic ethnicity persist for all three models. However, the magnitude of the odds ratios and the change in direction as compared with the individual race and ethnicity measures in Model 1 is of interest as it may suggest a population with high likelihood for missing their first appointment. In Model 2, the odds ratios and $p$-values for the demographic variables are generally similar to Model 1 . The addition of the payment type indicator adds additional explanatory value. Persons with private insurance are $28 \%$ less likely to miss the first appointment than patients who self-pay whereas publicly insured patients were $24 \%$ more likely to miss the first appointment. In Model 3 we add interaction terms for race by payment type. The odds ratios and significance levels of the Model 1 and 2 variables are similar to Model 3 with a couple of exceptions. Black race alone is no longer significant $(p>10)$ and the Black Hispanic interaction term is also no longer significant $(p>.10)$. However, we do see estimates of interest in the interaction terms for race by payment type added in Model 3. Black race with public private insurance have both have increased odds of a missed appointment relative to white patients who are "self-pay" $(\mathrm{O} . \mathrm{R} .=1.67$, $p=.04$, and O.R. $=2.51, p=.014)$ respectively. This contrasts with the small or non-significant effect observed for the Black race indicator in all models suggesting that the effect of Black race on missed first appointment condition is modified by insurance type. 
Table 1. Frequencies and percentages of the variables for the first visit analysis by appointment status $(n=1,850)$

\begin{tabular}{|c|c|c|c|c|c|c|}
\hline & \multirow{2}{*}{ Categories } & \multirow{2}{*}{ Frequency } & \multirow{2}{*}{ Percent } & \multicolumn{2}{|c|}{ Appointment Condition } & \multirow{2}{*}{$\begin{array}{l}p \text {-value for between } \\
\text { group test }\end{array}$} \\
\hline & & & & Kept & Missed & \\
\hline \multirow{2}{*}{ Age } & \multirow{2}{*}{ Continuous } & \multirow{2}{*}{ - } & \multirow{2}{*}{-} & $\mathrm{M}_{\mathrm{kept}}=46.25$ & $\mathrm{M}_{\text {miss }}=46.95$ & \multirow{2}{*}{$.374^{\xi}$} \\
\hline & & & & $S D_{\mathrm{kept}}=14.83$ & $S D_{\text {miss }}=14.44$ & \\
\hline \multirow{2}{*}{ Gender } & Female & 934 & 50.5 & 695 & 239 & \multirow{2}{*}{$.573^{\varphi}$} \\
\hline & Male & 916 & 49.5 & 692 & 224 & \\
\hline \multirow{3}{*}{ Race } & Black & 760 & 41.1 & 552 & 208 & \multirow{3}{*}{$.114^{\varphi}$} \\
\hline & White & 1005 & 54.3 & 767 & 238 & \\
\hline & Other & 85 & 4.6 & 68 & 17 & \\
\hline \multirow{2}{*}{ Ethnicity } & Hispanic & 67 & 3.6 & 51 & 16 & \multirow{2}{*}{$.825^{\varphi}$} \\
\hline & Non-Hispanic & 1783 & 96.4 & 1336 & 447 & \\
\hline \multirow{3}{*}{ Payment type } & Private & 245 & 13.2 & 198 & 47 & \multirow{3}{*}{$.009^{\varphi}$} \\
\hline & Public & 466 & 25.2 & 329 & 137 & \\
\hline & Self-Pay & 1139 & 61.6 & 860 & 279 & \\
\hline \multirow{2}{*}{ Marital Status } & Married & 467 & 25.2 & 353 & 114 & \multirow{2}{*}{$.722^{\varphi}$} \\
\hline & Not Married & 1383 & 74.8 & 1034 & 349 & \\
\hline Appointment & Kept & 1387 & 75.0 & & & \\
\hline Condition & Missed & 463 & 25.0 & & & \\
\hline
\end{tabular}

Note. ${ }^{\xi}$ Independent samples $t$-test for continuous age by appointment condition; ${ }^{\varphi}$ Pearson chi-square test by appointment condition

\section{Discussion}

Our retrospective study characterized all the patients who were recommended to a TCC during its first three years of operation. The study is unique because it provides information for an under-investigated vulnerable population, namely middle-aged, mostly non-insured disadvantaged patients lacking a medical home. With respect to the primary aim of this study, our hypothesis that Black race would be associated with lower likelihood of keeping the first appointment had some weak support in Model 1 and 2 but disappeared completely in Model 3. However, we did find that privately and publicly insured Black patients were at increased likelihood of a missed first appointment. Our hypothesis that uninsured patients would be less likely to keep the first appointment was not supported. However, we did learn that privately insured patients were more likely and publicly insured patients were less likely to keep their first TCC appointment. Our relatively small sample of Hispanics and persons of other race were too imprecise to have much weight however they may suggest populations potentially at risk for not keeping their first appointment.

Our finding is inconsistent with Elliot et al.'s 2016 study in Seattle, which showed a greater likelihood for minorities and the uninsured to keep their follow-ups with physicians. ${ }^{[8]}$ However, minorities in Seattle are ethnically very mixed as contrasted with northern Florida which has a predominantly African American and relatively small Hispanic minority population. The difference may imply that there are undetected factors in these two samples which may influence outcomes. According to Hawn, ${ }^{[15]}$ hospitals caring for the sickest, poorest, and most vulnerable patients were more likely to receive penalties from the Readmission Reduction Program due to their high RH rate. Uninsured and disadvantaged patients may benefit most by keeping their appointments if keeping a follow-up appointment for TC is a key step in preventing ER revisits or RH. ${ }^{[8]}$ Still, more needs to be characterized regarding the role of insurance status and vulnerable patients' and keeping initial appointments at TC programs. ${ }^{[7-13]}$

Our finding that white patients with private or public insurance exhibited very different likelihoods of keeping the first appointment than Black patients who were also insured. This accentuates differences between advantaged and disadvantaged populations that are likely linked with the social determinants of health. The role of private managed care companies and the strength of their physician networks for both public and private insurance need further investigation to determine if access to care is less than optimal among this population. Participants in this study referred to TC due to lack of a regular physician or inability to obtain an appointment within seven days of discharge were not distinguishable from patients referred for other reasons.

Elliot et al. ${ }^{[8]}$ revealed that the most disadvantaged patient subgroups (e.g., those who were homeless, had a history of substance abuse, or more frequent previous ER visits) were less likely to keep their follow-up appointments. More detailed information about the socioeconomic, medical and behavioral conditions of the current study population might 
shed light on reasons for kept versus missed appointments as would qualitative interviews with these most vulnerable patients. We speculate that some unmeasured factors in the current study may be predictive of kept versus missed appointments, e.g. social capital, transportation, or time constraints related to family responsibilities or multiple lowwage employment. ${ }^{[17]}$ It is also possible that patient misunderstanding about the purpose of the TC visit or its cost (none) may play a role.

This study as presented represents preliminary findings from an ongoing examination of this TC center. The analysis in this study was limited to simple bivariable relationships and regression models with small cell size for some variables of interest. Other limitations of the present study include its cross sectional nature and the use of a convenience sample. Findings from this regional sample cannot be generalized to the population outside of the area. The original appointment database did not include socioeconomic status (e.g., home- lessness) and behavioral health information (e.g., substance abuse) of patients, which could affect appointment status. Despite the limitations, our findings offer information from a large and complete sample of the first three years of a TC primarily serving an under-investigated population. Linking hospital data to TC appointment data is likely important for understanding the TC population.

Regarding implications for health disparity policies, our findings suggest that simply making a follow-up appointment may not guarantee its accomplishment at the TC for this type of disadvantaged patients. Medical teams need to collaborate with other health professionals, such as social workers, to identify the barriers to keeping appointments and ensure the effective solutions for achieving the goal of preventing future ER and RH utilization. This would include, but is not limited to, identifying a source of health insurance coverage for the most vulnerable patients.

Table 2. Nested logistic regression models by block

\begin{tabular}{|c|c|c|c|c|c|c|}
\hline \multirow{2}{*}{ Models for Appointment Condition } & \multicolumn{2}{|c|}{ Model 1} & \multicolumn{2}{|c|}{ Model 2} & \multicolumn{2}{|c|}{ Model 3} \\
\hline & Odds Ratio & $p$-value & Odds Ratio & $p$-value & Odds Ratio & $p$-value \\
\hline Age & 1.005 & .186 & 1.004 & .309 & 1.004 & .355 \\
\hline Gender Female & 1.062 & .577 & 1.061 & .586 & 1.060 & .594 \\
\hline Gender Male & - & - & - & - & - & - \\
\hline Race Black & 1.198 & .107 & 1.206 & .095 & 0.941 & .681 \\
\hline Race Other & 0.252 & .023 & 0.254 & .254 & 0.295 & .069 \\
\hline Race White & - & - & - & - & - & - \\
\hline Ethnicity Hispanic & 0.166 & .081 & 0.176 & .091 & 0.178 & .094 \\
\hline Ethnicity not-Hispanic & - & - & - & - & - & - \\
\hline Married & 0.977 & .853 & 0.979 & .868 & 0.977 & .857 \\
\hline Not Married & - & - & - & - & - & - \\
\hline Black Hispanic & 17.467 & .096 & 17.338 & .107 & 13.389 & .142 \\
\hline Other Hispanic & 35.542 & .004 & 31.724 & .005 & 31.988 & .006 \\
\hline White Hispanic & - & - & - & - & - & - \\
\hline Payment Private & - & - & 0.720 & .066 & 0.454 & .005 \\
\hline Payment Public & - & - & 1.239 & .090 & 1.026 & .874 \\
\hline Payment Self & - & - & - & - & - & - \\
\hline Black Public & & & & & 1.670 & .044 \\
\hline Black Private & & & & & 2.507 & .014 \\
\hline Black Self-Pay & - & - & - & - & - & - \\
\hline Other Public & & & & & 0.435 & .427 \\
\hline Other Private & & & & & -- & - \\
\hline Other Self-Pay & - & - & - & - & - & - \\
\hline White Public & - & - & - & - & - & - \\
\hline White Private & - & - & - & - & - & - \\
\hline White Self-Pay & - & - & - & - & - & - \\
\hline Observations (n) & & 1,850 & & 1,850 & & 1,850 \\
\hline -2 log-likelihood & & $2,061.6$ & & $2,053.29$ & & $2,041.78$ \\
\hline LR chi-sq. (dof) & M1 vs. M2 & $8.37(2)$ & M2 vs. M3 & $11.05(3)$ & M1 vs. M3 & $19.420(5)$ \\
\hline$p$-value & & .015 & & .012 & & .002 \\
\hline
\end{tabular}

Note. Model 1 demographics only; Model 2 demographics plus payment type; and Model 3 demographics, payment type and interaction terms for payment by race appointment status kept versus missed 


\section{FUNDING}

This study was funded by a grant from the Council on Research and Creativity, FSU Office of the Vice President for Research to Amy L. Ai, PhD. The opinion expressed in this article does not necessarily reflect the views of this organization.

\section{ACKNOWLEDGEMENTS}

We thank Nan Wang for his assistance as a research assistant in the early stages of this project.

\section{CONFlicts OF INTEREST Disclosure}

The authors declare they have no conflict of interest.

\section{REFERENCES}

[1] Sharma G, Fletcher KE, Zhang D, et al. Continuity of outpatient and inpatient care for hospitalized older adults. Jama the Journal of the American Medical Association. 2009; 301(16): 1671. PMid: 19383958. https://doi.org/10.1001/jama.2009.517

[2] Walraven CV, Oake BAN, Jennings MAA, et al. The association between continuity of care and outcomes: a systematic and critical review. Journal of Evaluation in Clinical Practice. 2010; 16(5): $947-$ 56. PMid: 20553366. https://doi.org/10.1111/j.1365-275 3.2009.01235.x

[3] Coleman EA, Boult C. American Geriatrics Society Health Care Systems, Improving the quality of transitional care for persons with complex care needs. J Am Geriatr Soc. 2003; 51(4): 556-7. https://doi.org/10.1046/j.1532-5415.2003.51186.x

[4] Krichbaum K. GAPN postacute care coordination improves hip fracture outcomes. West J Nurs Res. 2007; 29(5): 523-44. PMid: 17526868. https://doi.org/10.1177/0193945906293817

[5] Naylor MD. A decade of transitional care research with vulnerable elders. J Cardiovasc Nurs. 2000; 14(3): 1-14; quiz 88-9. PMid: 10756470. https://doi.org/10.1097/00005082-200004000 $-00004$

[6] Naylor MD, Aiken LH, Kurtzman ET, et al. The care span: The importance of transitional care in achieving health reform. Health Affairs. 2011; 30(4): 746-54. PMid: 21471497. https://doi.or g/10.1377/hlthaff.2011.0041

[7] Coleman EA, Min SJ, Chomiak A, et al. Posthospital care transitions: patterns, complications, and risk identification. Health Services Research. 2004; 39(5): 1449-66. PMid: 15333117. https: //doi.org/10.1111/j.1475-6773.2004.00298.x

[8] Elliott K, Klein JW, Basu A, et al. Transitional care clinics for followup and primary care linkage for patients discharged from the ED. American Journal of Emergency Medicine. 2016; 34(7): 1230. PMid: 27066931. https://doi.org/10.1016/j . ajem. 2016.03.029

[9] McDonagh JE, Kelly DA. The challenges and opportunities for transitional care research. Pediatr Transplant. 2010; 14(6): 688-700. PMid: 20557475. https://doi.org/10.1111/j.1399-3046.2010.0 1343. $\mathrm{x}$
[10] Naylor M, Keating SA. Transitional care: moving patients from one care setting to another. Am J Nurs. 2008; 108(9 Suppl): 58-63; quiz 63. PMid: 18797231. https://doi.org/10.1097/01. NAJ.000 $0336420.34946 .3 a$

[11] Naylor MD, Bowles KH, Brooten D. Patient Problems and Advanced Practice Nurse Interventions During Transitional Care. Public Health Nursing. 2000; 17(2): 94-102. PMid: 10760191. https: //doi.org/10.1046/j.1525-1446.2000.00094.x

[12] Naylor MD, Brooten DA, Campbell RL, et al. Transitional care of older adults hospitalized with heart failure: a randomized, controlled trial. Journal of the American Geriatrics Society. 2004; 52(5): $675-$ 84. PMid: 15086645 . https://doi.org/10.1111/j.1532-541 5.2004.52202.x

[13] Wong FK, Ho MM, Yeung S, et al. Effects of a health-social partnership transitional program on hospital readmission: a randomized controlled trial. Social Science \& Medicine. 2011; 73(7): 9609. PMid: 21839564 . https://doi .org/10.1016/j. socscimed. 2011.06 .036

[14] Centers for Medicare and Medicaid Service Medicare Program; Resvisions to payment policies under the physician fee schedule, DME face-to-face encounters, elimination of the requirement for termination of non-random prepayment complex medical review and other revisions for Part B CY 2013. Final Rule with Comment Period. Fed Regist. 2012; 77.

[15] Hawn MT. Unintended Consequences of the Hospital Readmission Reduction Program. Ann Surg. 2015; 261(6): 1032-3. PMid: 25647062. https://doi.org/10.1097/SLA. 00000000000011 50

[16] Tallahassee Memorial HealthCare, in Annual Report for Fiscal Year 2012. Tallahassee Memorial HealthCare: Tallahassee, FL; 2013. 40 p.

[17] Schoen C, Osborn R, Doty MM, et al. A survey of primary care physicians in eleven countries, 2009: perspectives on care, costs, and experiences. Health Affairs. 2009; 28(6): 1171-83. PMid: 19884491. https://doi.org/10.1377/hlthaff.28.6.w1171 